PoS $\quad \begin{aligned} & \text { PROCEEDINGS } \\ & \text { OF SCIENCE }\end{aligned}$

\title{
Searches for strong production of supersymmetry in the fully hadronic final states at CMS
}

\section{Myriam Schönenberger* on behalf of the CMS collaboration}

Eidgenoessische Technische Hochschule Zuerich ( $\mathrm{CH})$

E-mail: mschoene@cern.ch

We report on searches for new physics in events with one or more jets and missing transverse energy. The searches use proton-proton collision data recorded in 2016 by the CMS experiment at the LHC corresponding to an integrated luminosity of $35.9 \mathrm{fb}^{-1}$. The results are interpreted in terms of several simplified models of supersymmetry.

EPS-HEP 2017, European Physical Society conference on High Energy Physics

5-12 July 2017

Venice, Italy

${ }^{*}$ Speaker. 


\section{Introduction}

Searches for strongly produced supersymmetry (SUSY) in the fully hadronic final state are well motivated by the large production cross section of gluinos and squarks and their high branching fraction into jets. These make the all-jets final state the discovery channel at the energy frontier. The two analyses with the latest results are discussed: the searches are performed in multi-jet events with missing transverse energy exploiting the $\mathrm{M}_{T 2}$ [1] and the $\mathrm{H}_{T}^{\text {miss }}$ [2] variables.

\section{Search strategy}

Figure 1 shows two example diagrams of simplified models of SUSY. Light squark production (Fig 1 left), will give around 2 jets of which 0 have a b-flavor, gluino production coupling to top quarks on the other hand (Fig 1 right) will give around 12 jets of which 4 are expected to have b-flavor. It makes thus sense to bin in jet and b-jet multiplicity for optimal sensitivity to the various models considered. For a fully hadronic analysis a lepton veto is applied. Furthermore binning in the scalar sum of the jet transverse momentum, $H_{T}=\sum_{j e t s}\left|\overrightarrow{p_{T}}\right|$, gives sensitivity to the energy scale of the event. The neutralino in the final state will give rise to possibly very large missing transverse energy $\left(Z_{T}\right)$. To suppress backgrounds with similar topologies, bins are introduced in the discovery variables of the two analyses: the missing transverse hadronic energy, $H_{T}^{\text {miss }}=\left|\sum_{\text {jets }} \overrightarrow{p_{T}}\right|$, and the stransverse mass, $M_{T 2}=\min _{p_{T}^{\vec{c}(1)}+p_{T}^{\overrightarrow{c(2)}}=p_{T}^{\vec{m} i s s}}\left[\max \left(M_{T}^{(1)}, M_{T}^{(2)}\right)\right]$.
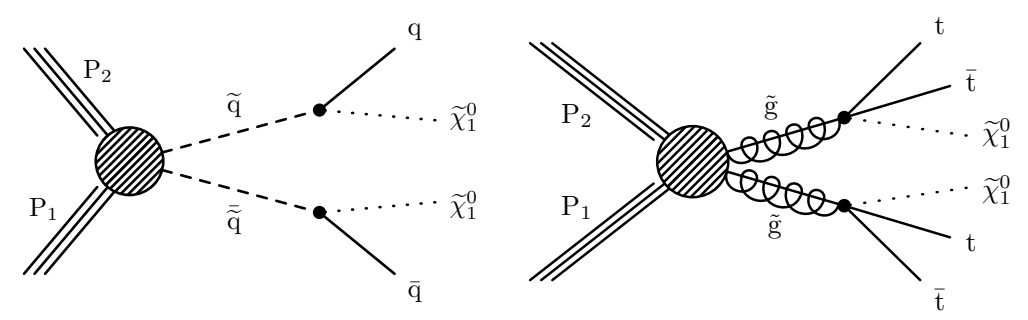

Figure 1: Example diagrams of simplified models of SUSY to illustrate the different final states: squark production to quarks (left) and gluino production to top squark pairs (right).

\section{Backgrounds}

The background composition in the all-jets final state is shown in Figure 2 for an $\mathrm{H}_{T}$ region of the $\mathrm{H}_{T}^{\text {miss }}$ analysis. QCD multi-jet events, shown in yellow, that are due to mismeasurement of a jet leading to an imbalance in the event, are strongly suppressed by kinematic selections. Top quark (blue) and $\mathrm{W}+$ jets (green) events contribute via leptonic decays of the $\mathrm{W}$ boson, where the event is not vetoed due to the lepton not being caught. The $\mathrm{Z}+$ jets background (red) from the $\mathrm{Z}$ boson decaying to two neutrinos is the most important background at low b-jet multiplicity. These background are discussed in the following. 


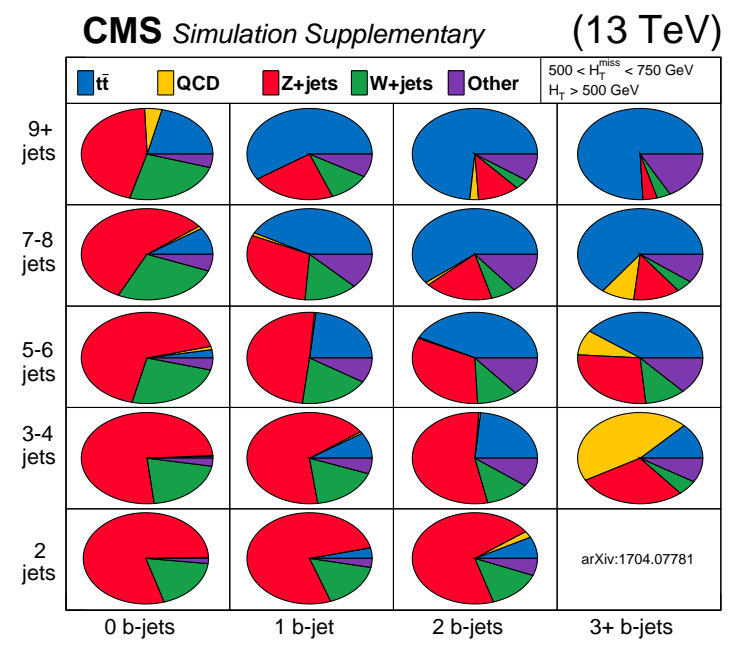

Figure 2: Background composition in bins of jet and b-jet multiplicity for $500 \mathrm{GeV}<\mathrm{H}_{T}<750 \mathrm{GeV}$ [2].

\subsection{QCD multi-jet estimate}

Multi-jet events have no intrinsic $B_{T}$, all missing energy is due to the detector response, which depends on the $p_{T}$ and $\eta$ of the jets, giving rise to instrumental $E_{T}$. In the rebalance and smear (R\&S) technique events are first adjusted by a likelihood minimization to the true hard scatter event with no $E_{T}$. These rebalanced events are then smeared with the response of the detector until all search regions are filled with an estimate. The closure test in simulation is show in Figure 3 (left), good agreement is observed between the standard simulation and the $R \& S$ estimate.
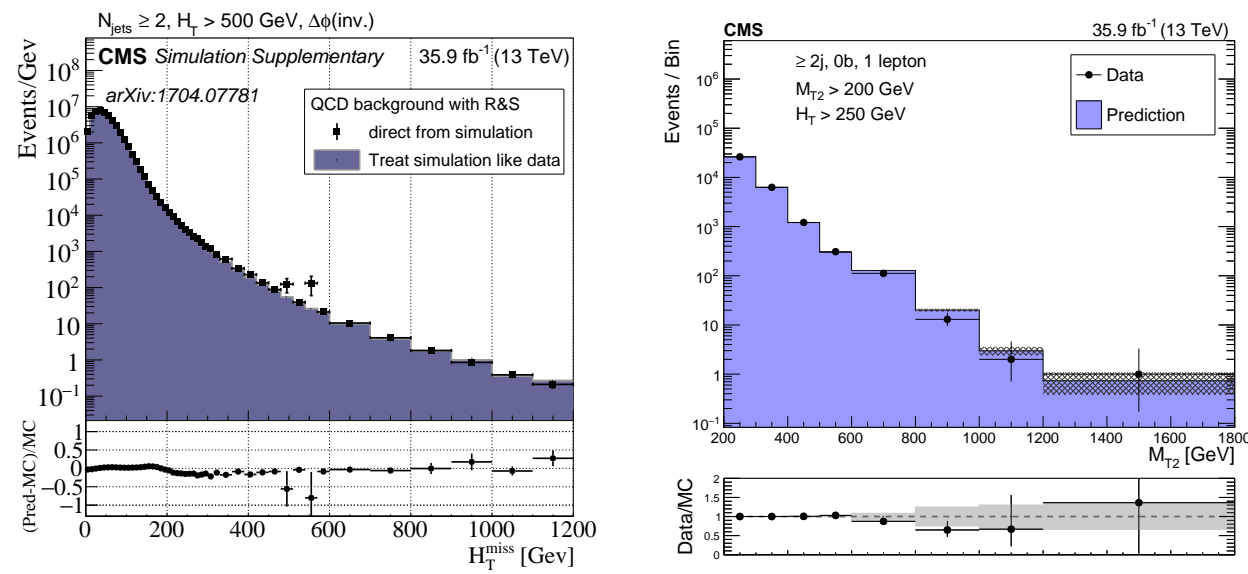

Figure 3: Closure test in simulation of the rebalance and smear method (left)[2]. Single lepton control region (right)[1].

\subsection{Lost lepton}

Events with leptonically decaying $\mathrm{W}$ bosons where the charged lepton is lost end up in the 
signal region. The lepton veto is designed to suppress events with charged leptons as effectively as possible, still the charged leptons can be missed because of the acceptance of the detector, the reconstruction and identification efficiency and non-isolation of the lepton, i.e. it being close to a jet. This background is estimated with a single lepton control region in data, shown in Figure 3 (right), and a transfer function taking into account the probability of losing a lepton in simulation.

Hadronic decays of tau leptons are treated by the $\mathrm{H}_{T}^{\text {miss }}$ analysis in a dedicated manner: a single muon control region in data is used to estimate this background. The $\mathrm{p}_{T}$ of the muon is smeared with the response function of the hadronic tau decays, then the event kinematics are recomputed. Figure 4 (left) shows the closure test of the method and good agreement between the standard simulation and the estimate are obtained.

\section{3 $\mathrm{Z}$ to invisible}

A $\mathrm{Z}$ boson decaying to two neutrinos is an irreducible background due to the similarity to the signal topology. Historically the way to estimate this background is to take a photon and remove it from the event to model the invisible decay of the $\mathrm{Z}$ boson. The advantage of this photon control region is its large statistical power, but it suffers from the theoretical uncertainty of the $Z / \gamma$ ratio and the uncertainty stemming from the production of fragmentation photons. Similarly one can use the leptonic decays of a $\mathrm{Z}$ boson to estimate the decays to neutrinos. In the same fashion as for the photons, the leptons are removed from the event to model the neutrinos. Due to the low branching fraction into leptons this method suffers from lower statistical power, and was in fact not feasible previously with lower collected integrated luminosity. It has the advantage though that it is essentially the same process and thus lower theoretical systematic uncertainties arise. Furthermore the opposite flavor di-lepton data control region allows for an estimate of the same flavor di-lepton contribution of top quark decays. Figure 4 shows the comparison for the $\mathrm{M}_{T 2}$ analysis of the different estimates compared to the $Z \rightarrow v v$ simulation.
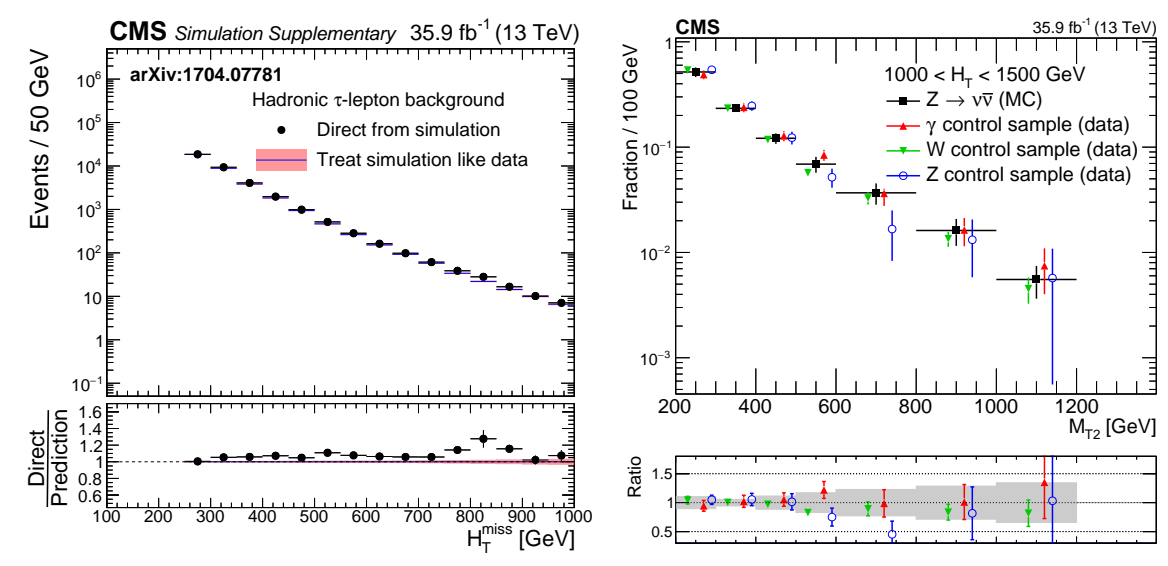

Figure 4: Closure test of the hadronic tau estimate (left)[2]. Comparison of the estimates from photons (red) and from $Z \rightarrow l l$ (blue) to the $Z \rightarrow v v$ simulation (black)(right)[1]. 


\section{Results}

Selected search regions are shown in Figure 5. No excess over the standard model predictions was observed. For easier reinterpretation of these results the covariance matrix of the search bins, see Figure 6, is provided along with aggregate signal regions, which are sums of bins that are particularly interesting for certain models, shown in Figure 6. These aggregate regions give worse limits though, from a factor of 1.1 up to a factor 3 worse than the full analysis [3].
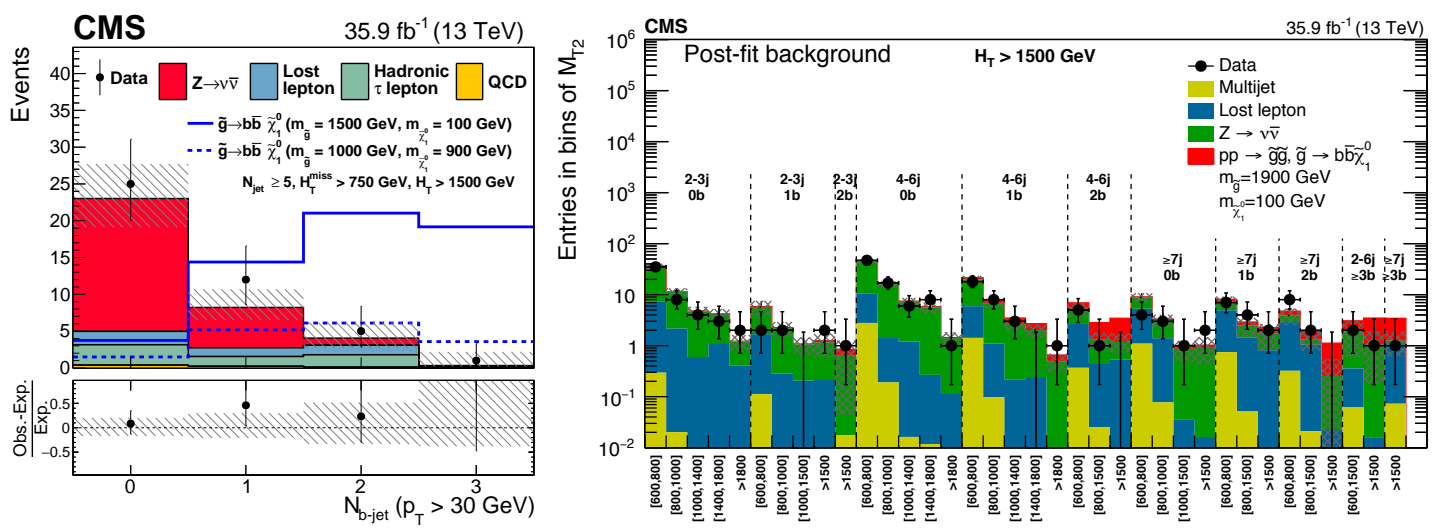

Figure 5: Selected results of the $\mathrm{H}_{T}^{\text {miss }}$ analysis (right)[2] and the $\mathrm{M}_{T 2}$ analysis(left)[1] with signals overlayed (blue lines, red histogram respectively).
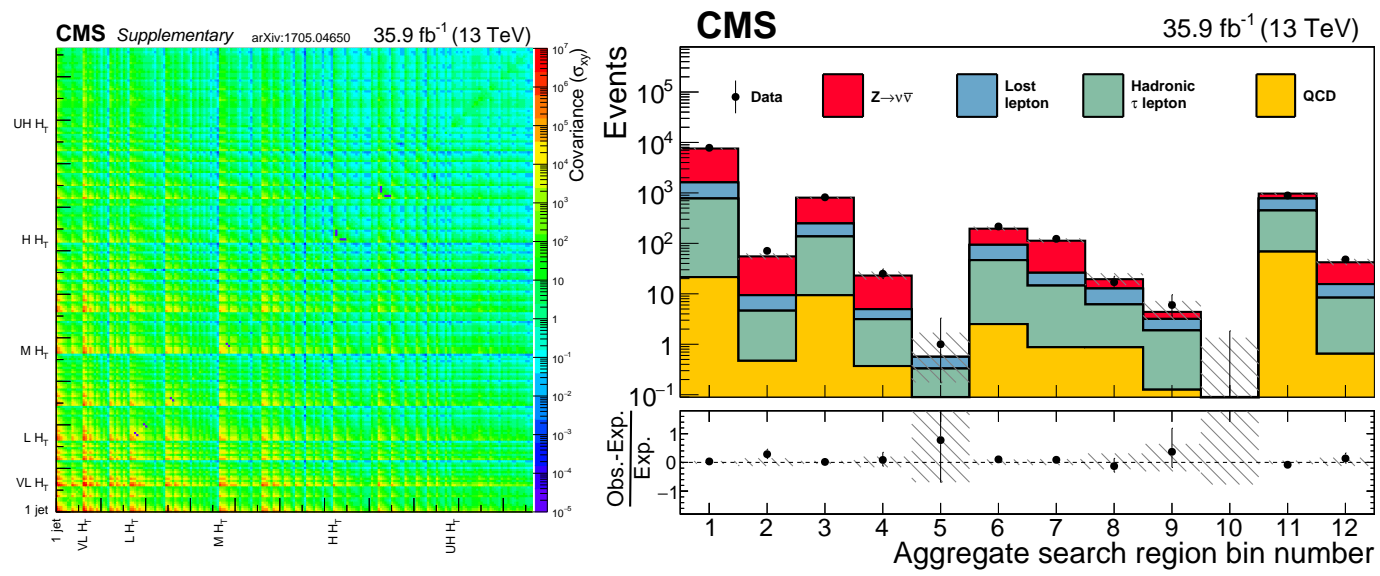

Figure 6: Covariance matrix of the $\mathrm{M}_{T 2}$ analysis (right)[1] and the aggregate regions of the $\mathrm{H}_{T}^{\text {miss }}$ analysis (left)[2].

\section{Interpretation}

Observed yields agree well with the expected SM backgrounds. The results are used to set upper limits on the SUSY production cross section in terms of simplified models. Figure 7 and 8 
show the limits for the two analyses for the gluino and squark production, respectively. Gluinos of roughly up to $2 \mathrm{TeV}$ and squarks of roughly up to $1 \mathrm{TeV}$ are excluded depending on the production model.
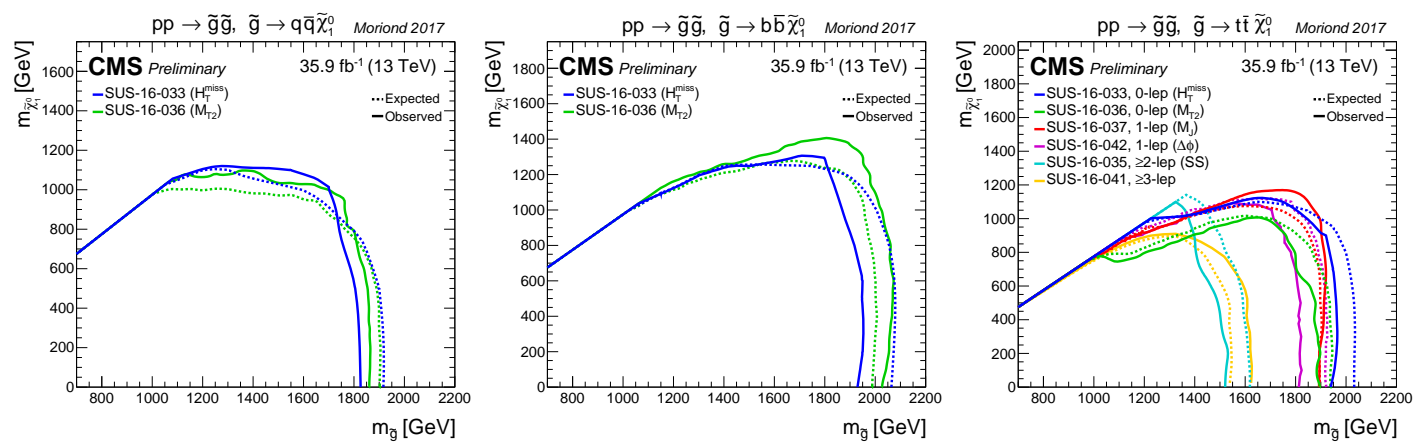

Figure 7: Upper limits on the gluino versus neutralino plane for gluino production coupling to light quark (left), bottom quark (middle) and top quark final states (right) for the $\mathrm{H}_{T}^{\text {miss }}$ analysis (blue) $\mathrm{M}_{T 2}$ and the analysis (green).
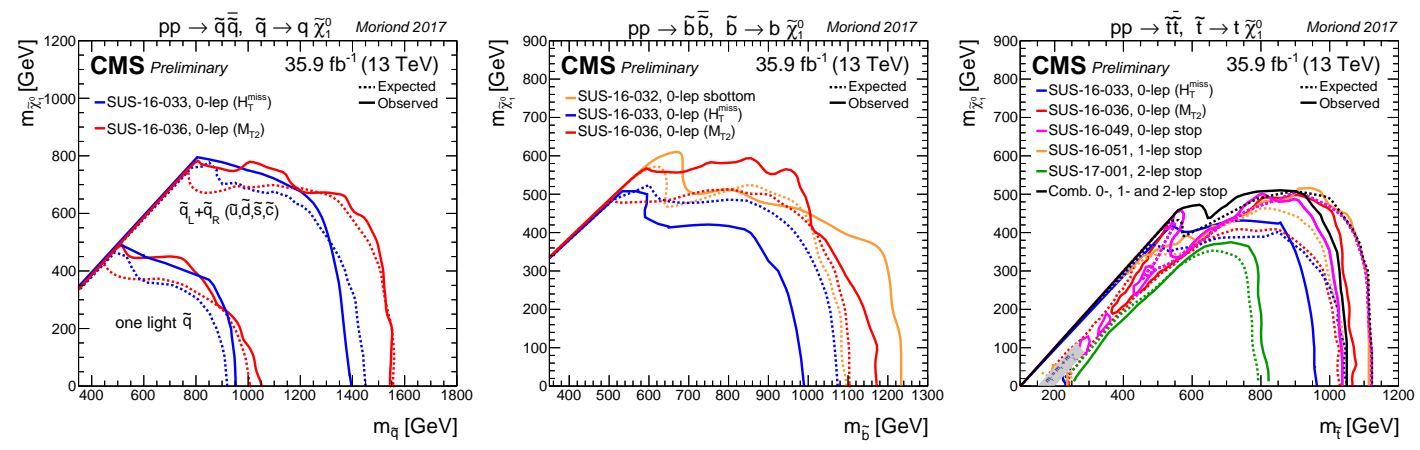

Figure 8: Upper limits on the squark versus neutralino plane for light squark (left), bottom squark (middle) and top squark production (right) for the $\mathrm{H}_{T}^{\text {miss }}$ analysis (blue) and the $\mathrm{M}_{T 2}$ analysis (green).

\section{Summary}

Searches for direct gluino and squark production in the hadronic final state have been presented. No sign of new physics has been found with $35.9 \mathrm{fb}^{-1}$ collected by the CMS detector. Exclusion limits are set on the masses of gluinos and squarks of up to roughly $2 \mathrm{Tev}$ and $1 \mathrm{TeV}$, respectively.

\section{References}

[1] CMS Collaboration. Search for new phenomena with the $\mathrm{M}_{\mathrm{T} 2}$ variable in the all-hadronic final state produced in proton-proton collisions at $\sqrt{s}=13 \mathrm{TeV}$. (CMS-SUS-16-036-003), May 2017. arXiv:1705.04650; Accepted by EPJC. 
[2] CMS Collaboration. Search for supersymmetry in multijet events with missing transverse momentum in proton-proton collisions at 13 TeV. Phys. Rev., D96(3):032003, 2017. arXiv:1704.07781.

[3] CMS Collaboration. Search for new physics in the all-hadronic final state with the $\mathrm{M}_{\mathrm{T} 2}$ variable. $C M S$ Physics Analysis Summary, (CMS-PAS-SUS-16-015), 2016. https://cds.cern.ch/record/2205162. 